\title{
Does ageing Singapore need an electronic database of hip fracture patients? The Value and role of a National Joint Registry and an electronic database of intertrochanteric and femoral neck FRACTURES
}

Singapore Med J 2014; 55(5): 287-288 doi:10.11622/smedj.2014072

It seems as if someone from the 'industry' knocks on our doors every week to tell us about 'the next best prosthesis for joint replacement or implant for fracture surgery' in orthopaedic surgery. How do we objectively evaluate the performance of these new implants while practising evidence-based medicine? It is important to remember that those who do not know the history of these new protheses are doomed to repeat it. Obviously, by recording all the events, mishaps and incidences of complications following the use of these prostheses or implants by various surgeons, and making that information readily available to other like-minded orthopaedic surgeons, will help them to be judicious in their selection of patients and prosthesis or implants. It is in this context that the value of a National Joint Registry (NJR) and Hip Fracture Registry (HFR) cannot be overstated. Not only does it assist in the short- and long-term follow-up of patients, it also "helps to monitor the performance of implants and the effectiveness of different types of surgery, improving clinical standards and benefiting patients, clinicians and the orthopaedic industry", as stated by the United Kingdom (UK) NJR. ${ }^{(1)}$

Data collection and registries are already established in several advanced Wvestern countries. Their value has been demonstrated by the early recognition of accelerated failure rate of the metal-on-metal Articular Surface Replacement (ASR) hip arthroplasty implant. ${ }^{(2)}$ Data collection and analysis from these different NJRs have raised concerns regarding metal-onmetal surface replacement and ensured that this information was publicised expeditiously throughout the orthopaedic community, thus ensuring patient safety and reducing risk to more patients. ${ }^{(3-6)}$ This in turn has improved clinical standards and reduced liability litigation, benefiting the industry.

Joint registries not only play a role, but have also "demonstrated value as a resource for the study of large numbers of patients, providing the opportunity to study rare occurrences and identify early failures of surgical procedures".(7) So why should we not develop our own national registries? It could be argued that it would be difficult to collate the required information. However, in 1998, National University Hospital (NUH) in Singapore introduced the use of electronic patient records in the form of the Computerized Patient Support System (CPSS). This was a significant milestone. Patient information, including patient history, previous admissions, current and past medications, as well as prior procedures and/or operations could be retrieved at the touch of a button. Shortly after CPSS went online, the Picture Archiving System (PACS) was introduced, complementing the former by making available all radiology images. Whilst these new technologies allowed rapid access to data, sped up clerical processes and improved overall patient care, as management decisions could be made in a timelier manner, they also contain the basic information required for National Joint and Hip Fracture Registries. Furthermore, by having expanded and integrating CPSS and PACS to involve all hospitals and polyclinics across Singapore, this ensured that any complication following arthroplasty or hip fracture surgery could be captured.

With so many registries worldwide, does Singapore really need one of its own? Can we not infer patient outcome by studying the existing registries from other countries? The United States of America has regional databases and registries. Australia, Norway, Iceland, Sweden and the UK all have NJRs, with Norway also having an HFR. However, they contain information predominantly on Caucasian patients. We have seen that these registries, particularly those for trauma implants, are designed for northern European patients and often do not suit the morphology of Asian bones. Thus, fracture implants have recently been redesigned to suit Asian patients. Having our own NJR and HFR would thus allow us to see how our population fares following surgery, and can be useful for future research and development of orthopaedic implants.

Currently, we already have tools available to develop our own registries, i.e. the CPSS and PACS. The UK, which only begun implementing electronic patient records last year, is experiencing teething problems, even though the country has had an NJR since 2002. We know that many hospitals in Singapore have 'stand-alone' registries of their own patients who have undergone arthroplasty or hip fracture fixation, using CPSS and PACS as the primary data source. NUH has developed the InterTrochanteric and electronic Neck Of Femur Fracture (IT eNOFF) database, where we can review our patient population admitted after sustaining a hip fracture from 2010 to 2012 inclusive. We identified 624 patients and reviewed 22 parameters, including patient demographics, mechanism of injury, type of injury and treatment. This information is readily accessible from the digitalised patient information systems. Within a short few weeks, we were able to achieve complete data acquisition for all parameters with perfect intra- and inter-observer reliability. Before the installation of integrated computer systems, data collection would have taken many months to complete.

Such databases are useful research tools. However, even though we can extrapolate some parameters for our hospital population, we are unable to draw conclusions or identify trends on a national basis, as the patient population varies from hospital to hospital. Furthermore, national planning in terms of health budgets and future resource requirements have become paramount 
during these times of fiscal restraint, and will become even more important with the expected increase in the aged population over the next couple of decades. According to the recently published Population White Paper, Singapore's total population could fall between 6.5 and 6.9 million by $2030 .{ }^{(8)}$ Whilst this figure has caused much concern, as highlighted in the Singapore media, more alarming is the fact that by 2030, 19\% of Singapore's population will be persons aged 65 years and above. ${ }^{(9)}$ The World Health Organization states that "approximately 1.66 million hip fractures occur each year worldwide" and "the incidence is set to increase four-fold by 2050 because of the increasing number of older people". ${ }^{10)}$ We feel that the potential for improving resource planning by having a much larger pool of patients in a National Joint and Hip Fracture Registry is self-evident. At present, several hospitals in the country already have individual databases. It would be more useful if these databases were interconnected and allowed sharing of information among various users, namely orthopaedic surgeons, hospitals, government departments, and perhaps, even the industry.

Are we prepared for the Singapore of the future? Can we accurately determine our needs? How good are we at identifying reasons for poor patient outcomes and failing implants? By building a National Registry now, we can plan and finance our future healthcare, and more importantly, meet the needs and demands of a growing, ageing population while continually striving to improve patient care. Therefore, we suggest culminating individual hospital data into a single National Joint and Hip Fracture Registry. Of course, there is a need to protect patients' privacy, especially with the introduction of the Personal Data Protection Act this year. This can be assured if a single, non-profit professional organisation, such as a national orthopaedic society, takes responsibility and control over access to the database. This will enable us to take advantage of the invaluable electronic data we possess, as well as assist in short- and long-term patient follow-up. In addition, it will allow us to infer patient outcomes for our own local population by studying our local registry rather than extrapolating data from other countries based on an incomparable patient population. As mentioned earlier, the potential for improving resource planning and patient care through the establishment of a large, single National Joint and Hip Fracture Registry is self-evident and warrants serious consideration.

Yours sincerely,

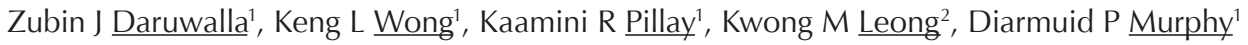

${ }^{1}$ Department of Orthopaedic Surgery, National University Hospital, Singapore, ${ }^{2}$ Medical Student, University of New South Wales, Australia. zubin_jimmy_daruwalla@nuhs.edu.sg

\section{REFERENCES}

1. National Joint Registry. About the NJR [online]. Available at: http://www.njrcentre.org.uk/njrcentre/AbouttheNJR/tabid/73/Default.aspx. Accessed November $28,2013$.

2. Langton DJ, Jameson SS, Joyce TJ, et al. Accelerating failure rate of the ASR total hip replacement. J Bone Joint Surg Br 2011; 93:1011-6.

3. Prosser GH, Yates PJ, Wood DJ, et al. Outcome of primary resurfacing hip replacement: evaluation of risk factors for early revision. Acta Orthop 2010; 81:66-71.

4. de Steiger RN, Miller LN, Prosser GH, et al. Poor outcome of revised resurfacing hip arthroplasty. Acta Orthop 2010; 81:72-6.

5. Corten K, MacDonald SJ. Hip resurfacing data from national joint registries: what do they tell us? What do they not tell us? Clin Orthop Relat Res 2010; 468:351-7.

6. Buergi ML, Walter WL. Hip resurfacing arthroplasty: the Australian experience. J Arthroplasty 2007; 22(7 Suppl 3):61-5. Review.

7. Maletis GB, Granan LP, Inacio MC, Funahashi TT, Engebretsen L. Comparison of community-based ACL reconstruction registries in the US and Norway. J Bone Joint Surg Am 2011; 93 Suppl 3:31-6.

8. National Population and Talent Division. A sustainable population for a dynamic Singapore: Population White Paper [online]. Available at: http://202.157.171.46/ whitepaper/downloads/population-white-paper.pdf. Accessed November 28, 2013.

9. Ministry of Social and Family Development. Briefing on ageing population in Singapore [online]. Available at: http://app.msf.gov.sg/Portals/0/Topic/lssues/ EDGD/Brief\%20on\%20Ageing\%20Population\%20(Public).pdf. Accessed November 28, 2013.

10. World Health Organization. Recommendations for preventing osteoporosis [online]. Available at: http://www.who.int/dietphysicalactivity/publications/ trs916/en/gsfao_osteo.pdf. Accessed November 28, 2013. 